\title{
Combining Multiple Seismic Attributes using Convolutional Neural Networks
}

\author{
Abrar Alotaibi ${ }^{1}$, Mai Fadel ${ }^{2}$, Amani Jamal ${ }^{3}$, Ghadah Aldabbagh ${ }^{4}$ \\ Computer Science Department, Faculty of Computing and Information Technology \\ King Abdulaziz University, Jeddah, Saudi Arabia ${ }^{1,2,3,4}$ \\ Computer Science Department, College of Computer Science and Information Technology \\ Imam Abdulrahman bin Faisal University, Dammam, Saudi Arabia ${ }^{1}$ \\ Department of Mechanical Engineering, Massachusetts Institute of Technology (MIT), Cambridge, MA, USA ${ }^{4}$
}

\begin{abstract}
Seismic exploration involves estimating the properties of the Earth's subsurface from reflected seismic waves then visualizing the resulting seismic data and its attributes. These data and derived seismic attributes provide complementary information and reduce the amount of time and effort for the geoscientist. Multiple conventional methods to combine various seismic attributes exist, but the number of attributes is always limited, and the quality of the resulting image varies. This paper proposes a method that can be used to overcome these limitations. In this paper, we propose using Deep Learning-based image fusion models to combine seismic attributes. By using convolutional neural network (CNN) capabilities in feature extraction, the resulting image quality is better than that obtained with conventional methods. This work implemented two models and conducted a number of experiments using them. Several techniques have been used to evaluate the results, such as visual inspection, and using image fusion metrics. The experiments show that the Image-fusion Framework, using the Image Fusion Framework Based on CNN (IFCNN) approach, outperformed all other models in both quantitative and visual analysis. Its $\mathrm{Q}_{\mathrm{AB} / \mathrm{F}}$ and $M S$-SSIM scores are $50 \%$ and $10 \%$, respectively, higher than all other models. Also, IFCNN was evaluated against the current state-of-the-art solution, Octree, in a comparative study. IFCNN overcomes the limitation of the Octree method and succeeds in combining nine seismic attributes with a better-combining quality, with $Q_{A B / F}$ and $N_{A B / F}$ scores being $40 \%$ higher.
\end{abstract}

Keywords-CNNs; neural networks; seismic attributes; seismic images; image fusion

\section{INTRODUCTION}

Seismic data is a major source of information for Earth subsurface exploration and visualization. To gather seismic data, seismic waves are sent into the Earth's subsurface, and the resulting reflection is recorded. Using these reflections, the underlaying structural information is obtained and the earth subsurface can be modeled and visualized [1]. The data that are obtained from the seismic data, to supplement and enhance the geological/geophysical information, are referred to as seismic attributes [2]. They help to make the process of visualization more informative.

The current process used by engineers, archeologists, geologists, and other scientific scholars to develop accurate representations of the Earth's subsurface involves looking at the seismic images and their related seismic attributes, which is followed by the interpretation of huge volumes of data. The process is, however, bulky and makes it difficult to combine the various views into one comprehensive view that can efficiently exploit all the data included in each individual view and reduce the time taken in the process.

Various scholars have made major contributions to address the challenge of combining seismic attributes, including Octree, principal component analysis (PCA), cross-plotting, and volume blending [3], [4]. The most recent work by AlDossari et al. [4], show how the Octree color quantization algorithm can be extended to enhance the combined seismic attributes. However, the method has some limitations. For instance, the number of attributes is limited to a maximum of eight, the structural disposition of the attributes can affect the results, and the result of the combined image includes artifacts.

Image fusion can be described as the process of combining more than one input image that contains complementary information from related scenes, thus producing a composite image [5]. The input images are obtained from matching imaging devices, including various types of imaging devices, or from various other parameters such as infrared cameras and satellites. The resultant composite image is more useful in terms of the included information as compared to the individual images [6]. The techniques used in image fusion offer many benefits in different image processing tasks that rely on viewing more than one image of the same scene, such as object recognition and detection, as well as areas like digital photography and remote sensing, among others. Merging the key information of various input images into one fused image can be helpful in reducing the challenge of wasted time and enhancing the final results of the work [5]. The data enrichment offered by seismic attributes of seismic images is the same as in various other image fusion tasks, like remote sensing and medical imaging.

The recent development of deep learning (DL) has led to various experts in the field developing different image fusion techniques using the new technology. In this field, Machine Learning algorithms, afforded by deep learning, along with neural networks, are used to extract data and image representations. The use of Convolutional Neural Networks (CNN) is important in solving the conventional, manual method challenge of designing fusion techniques and choosing 
activity-level metrics and fusion rules as it has the capability of learning features indirectly via data training. Because the tasks involved in image fusion are closely related to the classification challenges that $\mathrm{CNNs}$ excel in, they provide superior results [7], [8].

To create a DL method capable of combining any number of seismic attributes, this paper proposes using general image fusion models. The method involves extracting features of an image and then fusing them into a single image. It first obtains three-dimensional (3D) image information, with each piece of the three-dimensional information representing either the seismic attributes or the seismic (raw data) image. Based on this method, 3D data is sliced, and the resulting twodimensional (2D) images are forwarded to the fusion model as inputs. The key data is then extracted from the input images by use of the convolutional layer to produce maps of the features. These maps are then fused to generate the output image and, lastly, the process outputs the data in form of a $3 \mathrm{D}$ image.

This paper includes experiments that compared the proposed technique by implementing two fusion models with other fusion models used previously by Alotaibi et al. [9], and then compares their results. It also compares the model's results against the results of Octree. The models used are a new kind of image fusion model developed to fuse all types of images and are not limited to any specific types of images. The reason for using pre-trained models is the lack of available datasets for seismic images with ground-truth fusion images, which hinders the training process. The pretraining helps solve the problem of training the CNN.

Our paper is structured as follows: In Section II, we briefly provide background information; in Section III, the proposed fusion method is introduced in detail; in Section IV, the experimental results are shown; in Section V the conclusion of our paper and discussion are presented.

\section{BACKGROUND}

\section{A. Image Fusion Review}

In its simplest terms, image fusion can be described as a technique used in image processing that involves merging more than one input image, obtained from multiple sensors, to produce a single superior image [5]. The process is used to reduce the volume of data, as well as to provide images that are more ideal and understandable by computers and humans. Image fusion also facilitates the collection of data from images derived from multiple sources to create high-quality fused images including all the spectral and spatial information [6].

The fused image must observe the following conditions: first, it must contain all of the relevant information; second, it must have clarity regarding every artifact and anomaly; and third, all errors and noise are eliminated. Some of the primary applications of image fusion are multi-focus image fusion, medical image fusion, and remote sensing image fusion [5].

The common approach used in image fusion includes acquiring multiple input images, registering the images, and then fusing them. The registration of the images includes detecting features, setting and comparing them, estimating the transformation models, and converting and re-sampling the image. The process includes fusion rules which are applied either as part of the image transformation models or as direct mathematical applications, such as choosing or averaging the maximum pixel value [10].

Image fusion can be classified into different categories according to the task(s) performed [11]. These are:

- Multi-exposure image fusion - combines images with various exposures to different lighting to produce superior images.

- Medical image fusion - combines images used in medical fields, like computed tomography (CT) and magnetic resonance imaging (MRI) to produce more informative images.

- Infrared/Visible light image fusion that combines images obtained using infrared radiation with visible light to produce images that are more informative.

- Multi-focus image - it combines images that include diverse focus depths to produce greater depth of visual field.

The deep learning-based image fusion approach has demonstrated huge potential in terms of enhancing the techniques used for image fusion due to the application of CNNs. The basic architecture of CNNs includes two main parts, the classifier and the feature extractor. The latter utilizes pooling and convolutional layers to obtain the relevant features of the inputs and signify them via activation maps, which support the step of image registration in the image fusion process. The former is used to execute fusion rules on the map, which supports the fusion part in the image fusing process. CNNs are also able to apply more than one fusion rule since they are trained on big datasets, thus avoiding one of the classical limitations of the fusion techniques. Fig. 1 presents the CNN's basic architecture.

\section{B. General Image Fusion}

Within the last few years, a new trend of image fusion research has emerged in which DL models are created to perform image fusion on all types of image fusion tasks. So far, two of such general image fusion models have been developed.

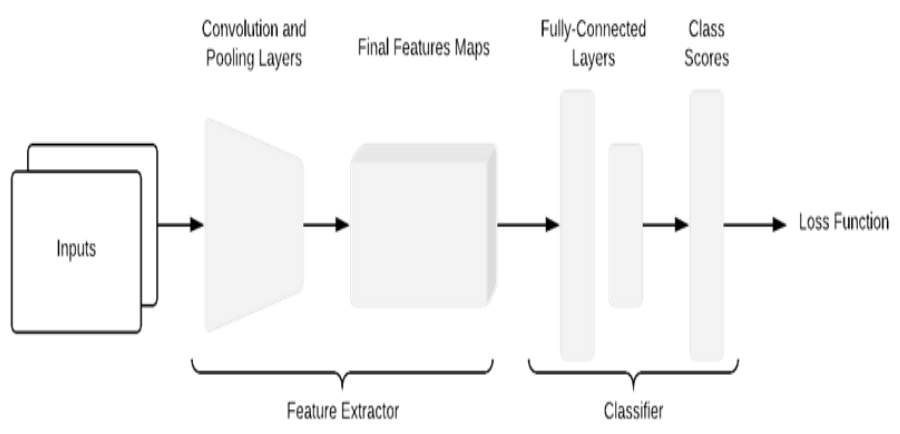

Fig. 1. Overview of CNNs Architecture. 
One of these includes a Zero-learning image fusion model proposed by Lahoud and Susstrunk [12] to solve problems of image fusion using CNNs, such as the need to create a large dataset to train the network. The training process is timeconsuming and expensive, requiring a lot of resources such as processing power, and a large amount of memory, energy, and time, which prevents the network from being able to perform other tasks. As a solution, the study proposes a novel image fusion model using earth CNNs. The pre-trained network is a network that has been trained on a large dataset and has saved the network's weights and biases to use on another task. Using a pre-trained network solves all the aforementioned problems by removing the need to create a dataset, saving time, and reducing cost. It also eliminates the training process to work on various image fusion tasks. The model operates as a twoscale decomposition image fusion model. Fig. 2, presents a schematic diagram of the proposed method. It follows specific steps, which include: (1) dividing the images into base and detail layers by applying a filter; (2) performing base layer fusion on saliency maps; (3) performing detail layer fusion on CNN feature maps using the Very Deep Convolutional Networks (VGG19) model trained with ImageNet [13]; and (4) fusing base and detail layers to acquire the final fused image.

The proposed model was tested against state-of-the-art models for medical image fusion, Infrared-Visible image fusion, and Multi-focus image fusion. The experimental results showed that the fusion model being developed was robust, and that it exceeded the current state-of-the-art image fusion models for specific tasks.

Zhang et al.[14] propose a general image-fusion framework using CNN (IFCNN) that takes full advantage of the convolutional layer capabilities as a feature extractor, as well as generating output images using a weighted average. The proposed framework is a novel solution to the problem of general-purpose image fusion to achieve state-of-the-art results with a fully convolutional neural network without the need for other techniques to complement it. The quality of the training dataset used in the model is far superior to other existing models, thus making the proposed framework a novelty among CNN models used for image fusion. The IFCNN contains three key modules, including the image reconstruction module, feature fusion module, and feature extraction module. Fig. 3 includes an illustration of the model architecture.

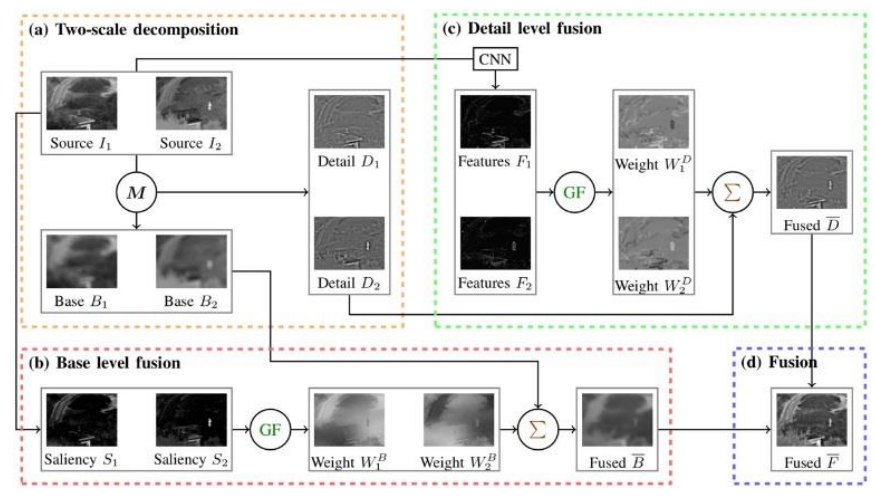

Fig. 2. Schematic Diagram of the Method [12].

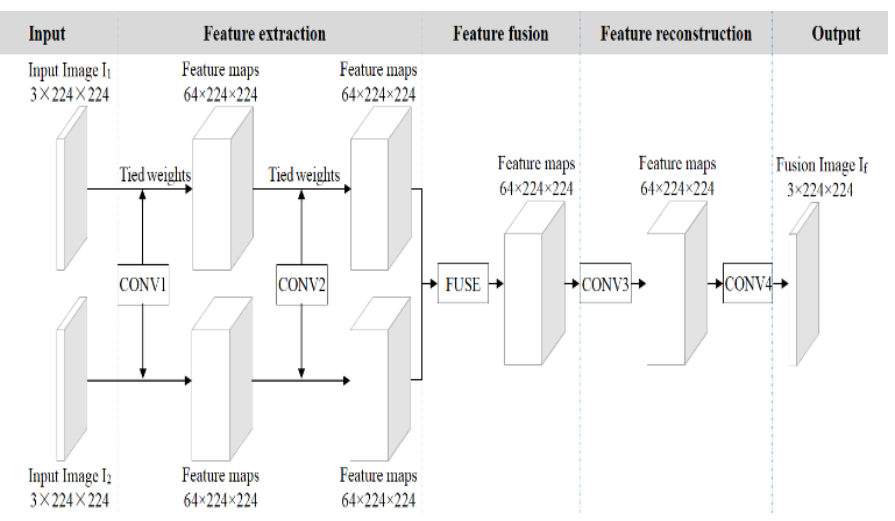

Fig. 3. IFCNN Architecture [14].

The model was also tested against state-of-the-art models used in multi-focus image fusion, multi-exposure image fusion, infrared-visible image fusion, and medical image fusion, and outperformed all of them.

Alotaibi et al. [9] proposed the use of DL models to combine seismic attributes. The work primarily investigated the performance of current state-of-the-art models, pre-trained for specific types of image fusion in combining three seismic attributes. The results showed that DeepFuse [15] has successfully combined three seismic attributes. DeepFuse is a model used for multi-exposure fusion. Prabhakar et al. [15] proposed DeepFuse, a novel model for multi-exposure fusion that takes an unsupervised approach in the fusion process. The authors also created and trained the network on a new benchmark dataset, improving the model's learning ability.

\section{PRoposed TECHNIQUE}

The image fusion technique proposed here helps to support the seismic data-merging and multiple seismic attributes along these lines: assume there are $\mathrm{X}$ inputs to the model, and $\mathrm{X} \geq 2$, where $\mathrm{X}$ is three-dimensional images with similar sizes that are either seismic attributes or data, symbolized as $I_{A n}$ and $I_{R}$ in that order, as $I_{\mathrm{An}} \mid \mathrm{n} \in\{1,2,3, \ldots, \mathrm{N}\}$ as shown in Fig. 4. First, the $I_{A n}$ and $I_{R}$ inputs are transferred to slicing functions to change the three-dimensional data $(\mathrm{x}, \mathrm{y}, \mathrm{z})$ into twodimensional data $(\mathrm{x}, \mathrm{y})$ with $\mathrm{Z}$ sum of images. The slicing function's outputs are sent to the fusion models as inputs, the model accept a group of images as inputs, including a single image from each $I_{A n}$ and $I_{R}$, beginning from $z=1$ up to $Z$. After every fused image is generated by the fusion models, and the calculation of the fusion metrics and image fusing is done, they are then converted into three-dimensional image information via the slicing function's reverse function.

\section{A. Fusion Model}

We will compare the performance of IFCNN and ZeroLearning models on the seismic image combining task. IFCNN is trained on a NYU-D2[16] dataset and ZeroLearning, using ImageNet weights for its layers, and pretrained implementation to overcome the issue of a lack of labeled datasets where their ground truths are identified. This approach also benefits from the use of pre-trained models because it eliminates the need for model training, which reduces the time and resources required to implement the method. Additionally, using pre-trained models to combine 
seismic attributes creates a method that is less complicated than all other existing methods since the existing methods require powerful workstations and high computational resources.

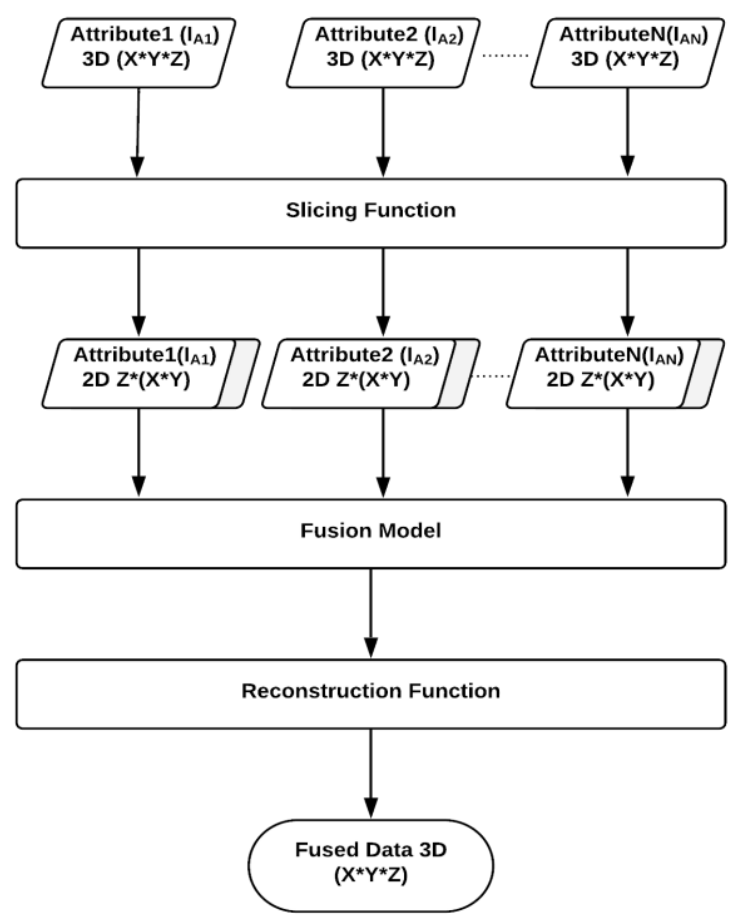

Fig. 4. Schematic Diagram of the Proposed Method.

\section{B. Fusion Metrics}

The metrics used to assess the performance of image fusion techniques proposed by Lahoud, Süsstrunk, et al. [12][14] compare the models' performance. Because the ground truth images are lacking, different non-referenced image fusion metrics are used in the evaluation of the performance of the models. Based on the study by Jagalingam and Hegde [17], the appropriate metrics to use are:

1) $\mathrm{N}_{\mathrm{AB} / \mathrm{F}}$ (Modified Fusion Artifacts): for measuring artificial artifacts produced by the fusion.

2) EN (Entropy): for measuring the fused image's information content.

3) $\mathrm{Q}_{\mathrm{AB} / \mathrm{F}}$ (Information Transfer): for measuring the overall information moved from source images to the fused one.

4) FMI (Feature Mutual Information): for measuring the dependencies existing between the fused image and input images.

5) MI (Mutual Information): measures the relationship of image intensity between the reference images and the fused images.

6) SSIM (Structural Similarity Index Measure): used for comparing the local patterns of pixel intensities between the reference images and the fused image.

7) MS-SSIM (Multi-scale structural similarity): used in measuring the expansion of the SSIM by merging luminance data at the highest resolution levels, with contrast and structural information at various down-sampled resolutions (scales).

\section{EXPERIMENTS AND RESULTS}

After conducting experiments on the models ZeroLearning and IFCNN, using pre-trained models published by Lahoud, Süsstrunk, et al. [12][14], the results were compared with those of DeepFuse and Octree [4][15]. Three key experiments are presented in this study. The first one compares the results from three models' results on combining three seismic attributes to find the best model, and the second one compares the results of Octree with the best fusion model.

Experiment 1 determined whether the proposed models are able to combine three different seismic images and then compared their results with DeepFuse. Experiment 2 showed the model's ability to combine up to nine attributes. Experiment 3 was used to compare the model results of combining eight seismic attributes against the results of Octree. To analyze the combined results, a visual comparison was done, along with a quantitative assessment, to check the visual representation characteristics, like color and quality, among other aspects of the fused image, together with the structural data.

\section{A. Comparing Fusion Models}

In the first experiment, a section from a marine block from the North Sea was used. The number of inputs $\mathrm{X}$ is 3 ; one of the inputs is a seismic image $\left(\mathrm{I}_{\mathrm{R}}\right)$, and another is a skeletonization algorithm seismic attribute termed skeleton, produced by $\left(\mathrm{I}_{\mathrm{A} 1}\right)$ [18]; the third is a seismic attribute called coherence and is represented by $\left(I_{A 2}\right)$ [19]. The size of $I_{R,}, I_{A 1}$, and $I_{A 2}$ is $(876,221,271)$. The inputs and the combined results of the three models are presented in Fig. 5. The images are reduced and cropped to fit within the limits of the space available. The original images were used for the experiments.

To assess the success of the fusion of multiple seismic attributes, the fusion result should:

1) Identify unique events that appear in one attribute. In this experiment, the events are faults that appear in one of the inputs.

2) Preserve small details.

3) Reveal major common geo-bodies from all inputs. In our experiment, the geo-bodies are faults [1]. (SP).

We will refer to each of these points as a Studied Property

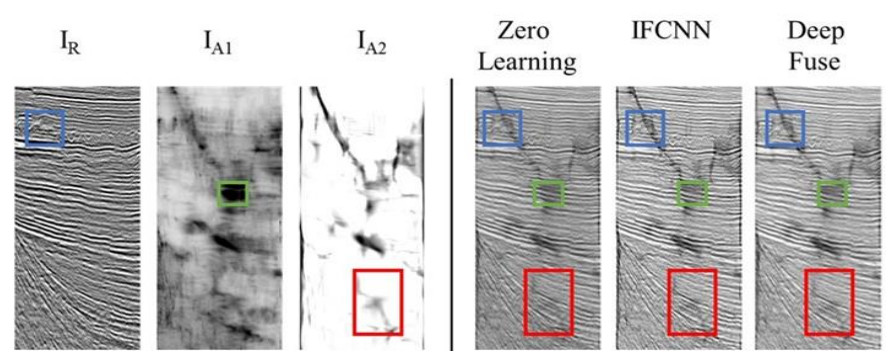

Fig. 5. Fusion Results after Combining Experiment 1 Results: The Left Side of the Line shows the Inputs while the Right Side Presents the Fusion Outputs. 
For SP 1, described as Identifying unique events that appear in one attribute, as shown in Fig. 4 and marked in blue, Zero-Learning, IFCNN, and DeepFuse clearly present the events from all inputs. For SP 2, described as Preserving small details after the combining process, as shown in Fig. 4 marked in red, IFCNN maintained all of the details that other models could not keep. For SP 3, described as Revealing major common Geo-bodies in the fused image, Fig. 4 shows an example of a common geo-body, marked in green. All models displayed the geo-body but not all details are visible. Only IFCNN captured all of the details. IFCNN had the best performance, followed by Zero-Learning, which shows that general fusion models are capable of extracting and transferring important information from seismic images better than DeepFuse.

Experiment 1 fusion metrics results are presented in Table I. The figures in bold font represent the best performance results, while underlined figures indicate the second-best performance.

TABLE I. EXPERIMENTAL RESULTS

\begin{tabular}{|l|l|l|l|}
\hline \multirow{2}{*}{ Fusion Metric } & \multicolumn{3}{|l|}{ Model } \\
\cline { 2 - 4 } & Zero Learning & IFCNN & DeepFuse \\
\hline $\mathrm{EN} \uparrow$ & $\underline{7.185351}$ & $\mathbf{7 . 3 6 3 6 4 6}$ & 7.127503 \\
\hline $\mathrm{MI} \uparrow$ & $\underline{21.55605}$ & $\mathbf{2 2 . 0 9 0 9 4}$ & 21.38251 \\
\hline $\mathrm{Q}_{\mathrm{AB} / \mathrm{F}} \uparrow$ & 0.35872 & $\mathbf{0 . 5 5 7 1 6}$ & $\underline{0.38529}$ \\
\hline $\mathrm{FMI} \uparrow$ & $\mathbf{0 . 8 4 0 9 9 6}$ & 0.831741 & $\underline{0.840794}$ \\
\hline $\mathrm{SSIM} \uparrow$ & $\mathbf{0 . 4 3 2 2}$ & 0.413706 & $\underline{0.428697}$ \\
\hline $\mathrm{MS}-\mathrm{SSIM} \uparrow$ & 0.785514 & $\mathbf{0 . 8 7 0 0 7 7}$ & $\underline{0.806129}$ \\
\hline $\mathrm{N}_{\mathrm{AB} / \mathrm{F}} \downarrow$ & $\underline{0.010318}$ & 0.079795 & $\mathbf{0 . 0 0 0 7 5 2}$ \\
\hline
\end{tabular}

The Zero-Learning model's result included the top values for FMI, SSIM, and second-best values for $\mathrm{EN}, \mathrm{MI}$, and $\mathrm{N}_{\mathrm{AB} / \mathrm{F} \text {. }}$ Scoring high in EN, MI, and FMI, $\approx 0.84$ values indicate that the fused images include huge volumes of data and that the model performs extremely well for extracting features. The model's EN and MI values are $2.4 \%$ less than the best model (IFCNN). Scoring high SSIM $\approx 0.43$, and MS-SSIM $\approx 0.78$ values, indicates that the fused images have maintained structural data and high image resolutions. The model's SSIM and MS-SSIM values are $1 \%$ and $9.7 \%$ respectively, less than the models with the highest values (IFCNN, DeepFuse). Scoring a high $\mathrm{Q}_{\mathrm{AB} / \mathrm{F}}, \approx 0.35$ value, shows that the fused image contains data transferred from the inputs. The model is ranked third for $\mathrm{Q}_{\mathrm{AB} / \mathrm{F}}$ value, and less than the best model by $35.6 \%$. Scoring a low $\mathrm{N}_{\mathrm{AB} / \mathrm{F}} \approx 0.01$ value indicates less artificial fusion noise. The model is ranked second for $\mathrm{N}_{\mathrm{AB} / \mathrm{F}}$ values and is more than the best model (DeepFuse) by $1 \times 10^{3}$ percent.

The IFCNN model's results included the top values in EN, MI, $\mathrm{Q}_{\mathrm{AB} / \mathrm{F}}$, and MS-SSIM. The model's high MI and EN values indicate that the fused image includes some rich information. The model has a high FMI $\approx 0.83$ value which is less than that of the model with best values (Zero-Learning) by $1 \%$. The model has a high SSIM $\approx 0.41$ and highest MS-
$\mathrm{SSIM} \approx 0.87$ values; SSIM values are $4.2 \%$ less than the best model (Zero-Learning). The model has the highest $\mathrm{Q}_{\mathrm{AB} / \mathrm{F}} \approx$ 0.55 value and has a low $\mathrm{N}_{\mathrm{AB} / \mathrm{F}} \approx 0.079$ value. It is ranked third for the $\mathrm{N}_{\mathrm{AB} / \mathrm{F}}$ values, and the value is more than the best model (DeepFuse) by $1 \times 10^{5}$ percent. The fused images contain all of the structural information from the inputs, every edge is clear, the inputs' texture and color are available, and no perceptible fusion noise is present.

For the DeepFuse model, its results included the top values for $\mathrm{N}_{\mathrm{AB} / \mathrm{F}}$, and the second-best ones for FMI, $\mathrm{Q}_{\mathrm{AB} / \mathrm{F}}, \mathrm{SSIM}$, and MS-SSIM. The model has high EN and MI values. The EN and $\mathrm{MI}$ values are $3.3 \%$ less than the model with the best EN and MI values (IFCNN) and the model's FMI $\approx 0.86$, and are less than the model with best values (Zero-Learning) by $0.02 \%$. The model has high SSIM $\approx 0.42$ and high MS-SSIM $\approx 0.80$ values. The SSIM and MS-SSIM values are $1 \%$ and $7.8 \%$ respectively, less than the models with the highest values (Zero-Learning and IFCNN). The model has a high $\mathrm{Q}_{\mathrm{AB} / \mathrm{F}} \approx 0.38$ value but is less than the best model (IFCNN) by \%30. The model has the lowest $\mathrm{N}_{\mathrm{AB} / \mathrm{F}} \approx 0.0004$ value. The fused images contain all of the structural information from the inputs, every edge is clear, the texture and color from the inputs are presented clearly, and no perceptible fusion noise is present.

The Experiment showed that IFCNN is the best out of the investigated models, as it outperformed every other model and gave the best result for enhancing fault detection by combining seismic attributes. Zero-learning and DeepFuse were competing for second place and had comparable performance. We only considered IFCNN in the following experiments.

\section{B. Combining more Attributes}

The second experiment tested IFCNN's ability to combine multiple seismic attributes. We used a section from the Parihaka dataset and generated fusion results. In addition to seismic Detect and Skeleton attributes, six additional attributes were generated using an edge-preserving algorithm [20]. Fig. 5 presents the results of combining multiple attributes.

The fusion metrics of the results have been calculated and are presented in Table II.

TABLE II. FUSION METRICS' RESULTS FOR IFCNN FOR UP TO NINE INPUTS

\begin{tabular}{|l|l|l|l|l|l|l|l|}
\hline \multirow{2}{*}{$\begin{array}{l}\text { Fusion } \\
\text { Metric }\end{array}$} & \multicolumn{6}{|l|}{ Number of Inputs (Attributes) } \\
\cline { 2 - 8 } & $\mathbf{3}$ & $\mathbf{4}$ & $\mathbf{5}$ & $\mathbf{6}$ & $\mathbf{7}$ & $\mathbf{8}$ & $\mathbf{9}$ \\
\hline$E N \uparrow$ & 6.85 & 6.86 & 6.89 & 7.02 & 7.14 & 7.06 & 7.60 \\
\hline$M I \uparrow$ & 20.9 & 27.4 & 34.48 & 42.13 & 50.00 & 56.55 & 60.87 \\
\hline$Q_{A B / F} \uparrow$ & 0.56 & 0.48 & 0.36 & 0.32 & 0.31 & 0.25 & 0.21 \\
\hline$F M I \uparrow$ & 0.86 & 0.84 & 0.84 & 0.82 & 0.82 & 0.82 & 0.76 \\
\hline$S S I M \uparrow$ & 0.50 & 0.43 & 0.41 & 0.44 & 0.43 & 0.38 & 0.28 \\
\hline $\begin{array}{l}M S- \\
S S I M \uparrow\end{array}$ & 0.85 & 0.69 & 0.60 & 0.73 & 0.55 & 0.70 & 0.67 \\
\hline$N_{A B / F} \downarrow$ & 0.05 & 0.05 & 0.01 & 0.04 & 0.13 & 0.08 & 0.17 \\
\hline
\end{tabular}


The metrics values show that IFCNN had maintained good image quality for the fused images while increasing the number of attributes. First, the increase in EN and MI values with the increase of attributes shows that IFCNN combining results is rich in information. IFCNN maintained high FMI and MS-SSIM values while increasing the number of attributes, demonstrating that the fused images did not lose important information from individual inputs and that the fused images have a good structure. The $\mathrm{N}_{\mathrm{AB} / \mathrm{F}}$ values increased while increasing the number of attributes, since increasing the number of inputs leads to increasing the amount of resulting fusion noise, but the values remained small. Finally, IFCNN $\mathrm{Q}_{\mathrm{AB} / \mathrm{F}}$ and SSIM values decreased while increasing the number of attributes because, given that the fused image structural similarity to individual inputs and the information transfer rate from input to output will decrease with the increase of inputs, the decrease is to be expected.

Visually inspecting the combining results exhibit IFCNN's ability to increase the number of combined attributes without generating a large number of unwanted artifacts or diminishing visual information. After examining the combining results quantitatively using fusion metrics, and qualitatively by visual inspection, the combining results determined IFCNN's ability to successfully combine up to nine attributes, with the ability to combine more.
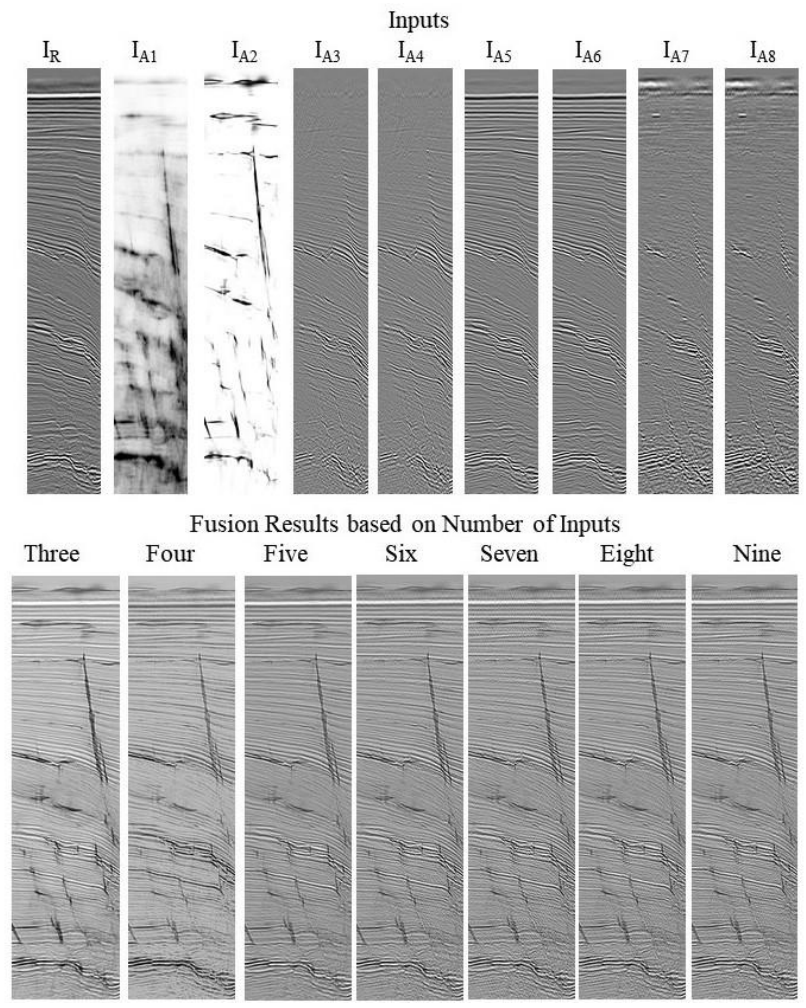

Fig. 6. A Sample of Combining Results of Multiple Attributes. Inputs: Seismic (IR), Detect Attribute (IA1), Skeleton Attribute (IA2), Sobel X Gradient Attribute (IA3), Prewitt X Gradient Attribute (IA4), Sobel Y Gradient Attribute (IA5), Prewitt Y Gradient Attribute (IA6), Sobel Z Gradient Attribute (IA7), Prewitt Z Gradient Attribute (IA8).

\section{Comparing IFCNN and Octree}

The third experiment was carried out to compare, in detail, the quality of combining results between IFCNN and Octree on fault detection. To fairly compare IFCNN and Octree, we used sections from a marine block and an F3 block to generate combining results of three and eight attributes respectively, Fig. 6 shows the input and output of both IFCNN and Octree for combining three attributes.

As shown in Fig. 6, IFCNN preserved more structural information in the resulting image than Octree, its combined image had less noise, and its results are more suited for the fault detection task. Then, we generated combining results for eight attributes and compared the performance of the two methods as shown in Fig. 8.

From Fig. 7, it can be seen that IFCNN's structural details are more vivid and that faults are more easily detectable since IFCNN uses a large number of filters to extract important features from individual attributes before combining. This helped IFCNN maintain high structural information with the increased number of attributes. The quality of the combining results can be quantified using the fusion metrics' values in Table III (better results are in bold).

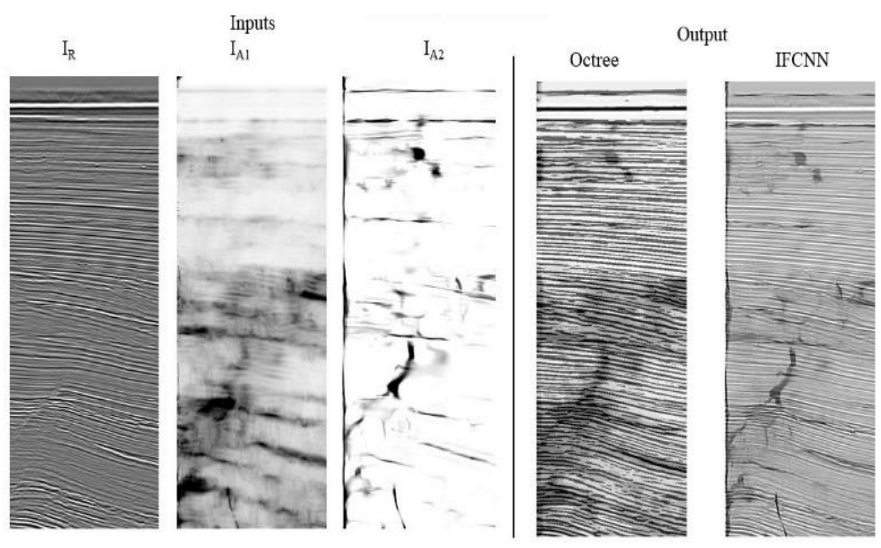

Fig. 7. Inputs: Seismic (IR), Detect Attributes (IA1), and Skeleton Attribute (IA2). Output: Combining Results of IFCNN and Octree.
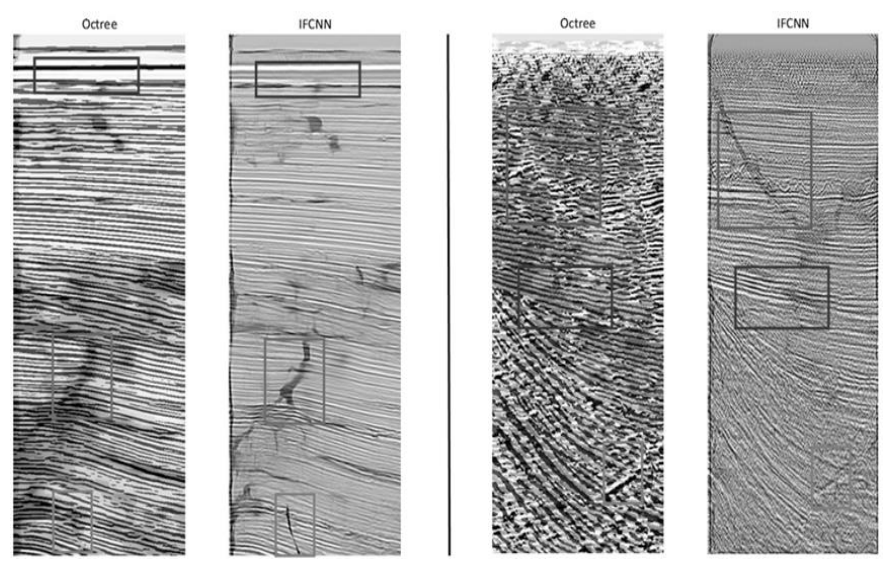

Fig. 8. Left Side: Combining Results of Three Attributes. Right Side: Combining Results of Eight Attributes. 
TABLE III. FUSION METRICS’ RESULTS FOR IFCNN AND OCTREE

\begin{tabular}{|l|l|l|l|l|}
\hline \multirow{2}{*}{ Fusion Metric } & \multicolumn{2}{|l|}{ 3 Attributes } & 8 Attributes \\
\cline { 2 - 5 } & IFCNN & Octree & IFCNN & Octree \\
\hline$E N \uparrow$ & 7.180216 & $\mathbf{7 . 5 1 1 5 6}$ & 7.60924 & $\mathbf{7 . 9 3 2 8 8}$ \\
\hline$M I \uparrow$ & 21.54065 & $\mathbf{2 2 . 5 3 4 7}$ & 60.8739 & $\mathbf{6 3 . 4 6 3 0}$ \\
\hline$Q_{A B / F} \uparrow$ & $\mathbf{0 . 5 8 3 8 2 6}$ & 0.27468 & $\mathbf{0 . 2 1 1 1 4}$ & 0.11362 \\
\hline$F M I \uparrow$ & 0.850726 & $\mathbf{0 . 8 7 9 1 3}$ & $\mathbf{0 . 7 6 6 4 8}$ & 0.72074 \\
\hline$S S I M \uparrow$ & $\mathbf{0 . 4 5 7 9 2 5}$ & 0.25939 & $\mathbf{0 . 2 8 0 5 6}$ & 0.18298 \\
\hline$M S-S S I M \uparrow$ & $\mathbf{0 . 8 7 8 8 0 3}$ & 0.41374 & $\mathbf{0 . 6 7 6 7 7}$ & 0.26245 \\
\hline$N_{A B / F} \downarrow$ & $\mathbf{0 . 0 5 6 3 7 7}$ & 0.35625 & $\mathbf{0 . 1 7 0 1 2}$ & 0.29739 \\
\hline
\end{tabular}

The higher EN and MI values of the Octree method can be attributed to the color quantization algorithm, because Octree quantizes and inputs pixel values, which guarantees that all information from all inputs is condensed in the output, creating an information-rich image with a high amount of information. The FMI values are also affected by the color quantization algorithm, as observed in Table III. The Octree method had a higher value of FMI with a small number of inputs because the total amount of information can be quantized (condensed) without losing input information, but when quantizing a large number of inputs, some information from individual inputs is lost. Because of feature extraction, IFCNN managed to maintain high values of FMI with the increase of the number of inputs, because the important information is extracted from the images, and the fused image did not lose important information from individual inputs. Also, for the other metrics, it can be seen that the Octree method does not maintain high structural similarities between inputs and output, which is indicated by the low values of SSIM and MS-SSIM. Fig. 7 shows that the Skeleton attribute structure is not clearly visible in the Octree results. IFCNN, SSIM, and MS-SSIM values are 50\% more than Octree, and this can be observed by IFCNN results maintaining a similar structure as input images. Also, IFCNN, $\mathrm{Q}_{\mathrm{AB} / \mathrm{F}}$, and $\mathrm{N}_{\mathrm{AB} / \mathrm{F}}$ values are $40 \%$ better than Octree, indicating the IFCNN causes less artificial noise during the combining, and it has a better information transfer rate than Octree. Thus, results show that IFCNN overcomes the limitations of the Octree method, and IFCNN's seismic attribute combination quality exceeds that of the Octree method.

\section{CONCLUSION}

In this paper, a method based on Deep Learning, designed to solve the problem of combing multiple seismic attributes by using CNN models is presented. The proposed method uses pre-trained general image fusion models to fuse and combine multiple seismic attributes. The approach has shown that it can:

1) Overcome the issue of lack of labeled datasets where their ground truths are identified.

2) Reduce the time and resources requirements by the use of pre-trained models to eliminate the training phase.

3) Provide a refined solution that is capable of producing better results than using the DeepFuse model.
The experiments that were conducted led to a number of findings. They showed that the Image-fusion Framework using the CNN (IFCNN) model was the best model out of all of the investigated models for combining seismic attributes. The Zero-Learning model is a lightweight model, and the easiest one to modify, and can be extended for future research to further study these types of fusion models to use for seismic attribute combining tasks. General image fusion models exhibited excellent results and showed great potential. The results showed that IFCNN scored better than other DL models on multiple metrics. Its $Q_{A B / F}$ and $M S$-SSIM scores are $50 \%$ and $10 \%$, respectively, higher than the second-best model. When IFCNN is compared to the current state-of-theart, Octree, IFCNN combining quality was superior to Octree especially when the number of attributes is high. Metrics results showed that IFCNN is better the Octree by $40 \%$ when the two are compared by the quality of the image structural information and the amount of noise.

The work presented in this paper implemented a method that can combine seismic attributes using pre-trained DL models. The choice of the pretrained models was based on their performance in comparison to other models found during the literature review. With scientific research advancement, better models can be introduced. The downside to the study presented is that the work is limited to seismic attributes used for faults enhancement and detection.

\section{ACKNOWLEDGMENT}

We would like to extend special thanks of gratitude to Dr. Saleh Aldossary for this golden opportunity to work on this project. We acknowledge and appreciate his unrelenting effort in advising us and providing test data for the project.

\section{REFERENCES}

[1] Al-Shuhail, S. A. Al-Dossary, and W. A. Mousa, Seismic Data Interpretation using Digital Image Processing. 2017.

[2] E. L. Galvan Aguilar, "Log property mapping guided with seismic attributes," Sep. 2013.

[3] S. S. Manral and D. Clark, "Multi Attribute Analysis-An effective visualization \& interpretation technique," 2010.

[4] S. Al-Dossary, J. Wang, and Y. E. Wang, "Combining multiseismic attributes with an extended octree quantization method," Interpretation, 2019, doi: 10.1190/int-2018-0099.1.

[5] B. Meher, S. Agrawal, R. Panda, and A. Abraham, "A survey on region based image fusion methods," Inf. Fusion, vol. 48, pp. 119-132, 2019, doi: 10.1016/j.inffus.2018.07.010.

[6] B. Ashalatha and M. B. Reddy, "Image fusion at pixel and feature levels based on pyramid imaging," in 2017 IEEE International Conference on Smart Technologies and Management for Computing, Communication, Controls, Energy and Materials, ICSTM 2017 - Proceedings, 2017, pp. 258-263, doi: 10.1109/ICSTM.2017.8089164.

[7] S. Li, J. T. Kwok, and Y. Wang, "Multifocus image fusion using artificial neural networks," Pattern Recognit. Lett., vol. 23, no. 8, pp. 985-997, 2002, doi: https://doi.org/10.1016/S0167-8655(02)00029-6.

[8] S. Li, X. Kang, L. Fang, J. Hu, and H. Yin, "Pixel-level image fusion: A survey of the state of the art," Inf. Fusion, vol. 33, May 2016, doi: 10.1016/j.inffus.2016.05.004.

[9] A. Alotaibi, M. Fadel, A. Jamal, and G. Aldabbagh, "Enhancement of 3D Seismic Images using Image Fusion Techniques," Int. J. Adv. Comput. Sci. Appl., vol. 12, no. 1, pp. 365-372, 2021, doi: 10.14569/IJACSA.2021.0120143.

[10] E. Blasch, Y. Zheng, and Z. Liu, Multispectral Image Fusion and Colorization. 2018. 
[11] Y. Liu, X. Chen, Z. Wang, Z. J. Wang, R. K. Ward, and X. Wang, "Deep learning for pixel-level image fusion: Recent advances and future prospects," Inf. Fusion, vol. 42, no. September 2017, pp. 158-173, 2018, doi: 10.1016/j.inffus.2017.10.007.

[12] F. Lahoud and S. Süsstrunk, "Fast and Efficient Zero-Learning Image Fusion," pp. 1-13, 2019, [Online]. Available: http://arxiv.org/abs/1905.03590.

[13] K. Simonyan and A. Zisserman, "Very Deep Convolutional Networks for Large-Scale Image Recognition," arXiv 1409.1556, Sep. 2014.

[14] Y. Zhang, Y. Liu, P. Sun, H. Yan, X. Zhao, and L. Zhang, "IFCNN: A general image fusion framework based on convolutional neural network," Inf. Fusion, vol. 54, no. July 2019, pp. 99-118, 2020, doi: 10.1016/j.inffus.2019.07.011.

[15] K. R. Prabhakar, V. S. Srikar, and R. V Babu, "DeepFuse: A Deep Unsupervised Approach for Exposure Fusion with Extreme Exposure Image Pairs," in 2017 IEEE International Conference on Computer Vision (ICCV), 2017, pp. 4724-4732, doi: 10.1109/ICCV.2017.505.

[16] H. Yan, X. Yu, Y. Zhang, S. Zhang, X. Zhao, and L. Zhang, "Single Image Depth Estimation With Normal Guided Scale Invariant Deep Convolutional Fields," IEEE Trans. Circuits Syst. Video Technol., vol. 29, no. 1, pp. 80-92, 2019, doi: 10.1109/TCSVT.2017.2772892.

[17] P. Jagalingam and A. V. Hegde, "A Review of Quality Metrics for Fused Image," Aquat. Procedia, vol. 4, pp. 133-142, 2015, doi: https://doi.org/10.1016/j.aqpro.2015.02.019.

[18] K. Vasudevan, D. Eaton, and F. A. Cook, "Adaptation of seismic skeletonization for other geoscience applications," Geophys. J. Int., vol. 162, no. 3, pp. 975-993, Sep. 2005, doi: 10.1111/j.1365246X.2005.02704.X.

[19] Y. Luo, W. G. Higgs, and W. S. Kowalik, "Edge detection and stratigraphic analysis using 3D seismic data," in SEG Technical Program Expanded Abstracts 1996, Society of Exploration Geophysicists, 1996, pp. 324-327.

[20] J. L. Gómez and D. R. Velis, "Structure-oriented edge-preserving smoothing in the frequency domain: Application to enhance 3D seismic data volumes," in 2017 XVII Workshop on Information Processing and Control (RPIC), 2017, pp. 1-5, doi: 10.23919/RPIC.2017.8211614.

\section{AUTHORS' PROFILE}

Abrar Alotaibi is a postgraduate student in the Computer Science Department, Faculty of Computing and Information Technology at King Abdulaziz University. She received a bachelor's degree from Imam Abdulrahman Bin Faisal University, Dammam, Saudi Arabia, in 2015. She holds a Teaching Assistant position at the College of Computer Science and Information Technology in the Computer Science Department at Imam Abdulrahman Bin Faisal University. Her research interests are Image Processing and Machine Learning. She is a member of IEEE.

Dr. Mai A Fadel is a lecturer in Software Engineering at the Department of Computer Science, King Abdulaziz University. Her research interest lies in
Design Patterns, and software engineering in general, cloud computing and news credibility in social networks and High-Performance Computing (HPC). She has taught courses in software engineering, web development, algorithms and Java programming. Dr. Mai was the Head of the Information System and the Computer Science departments between 2009 and 2016. Dr. Mai received her $\mathrm{PhD}$ degree in Computer Science from the Department of Computer Science at the University of Exeter, UK. She is a member of IEEE and ACM.

Dr. Amani Tariq Jamal is an assistant professor at the Computer Science Department, Faculty of Computing and Information Technology at King Abdulaziz University. She received her Master and Ph.D. degrees from Concordia University, Montreal, Canada, in 2009 and 2015 respectively. Her research interests are pattern recognition, artificial intelligence, Natural Language Processing, and document analysis. She is the cofounder and member of the directors' board of Saudi AI Society.

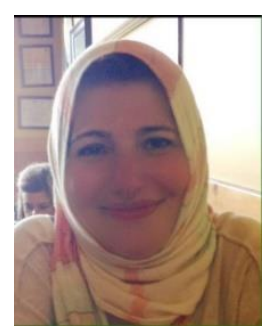

Dr. Ghadah Aldabbagh received her BSc in 1991 in Computer Science, from the College of Engineering at the University of Illinois at Urbana Champaign, Illinois, United States; she received her MSc in the Data Communication Networks and Distributed System (DCNDS) from the Computer Science Department at the University College London (UCL) in the United Kingdom; received her PhD in 2010 from the Department of Electronic and Electrical Engineering at UCL, UK. Dr. Aldabbagh holds an Associate Professor position at the Computer Science Department at the Faculty of Computing and Information Technology (FCIT) at King Abdul Aziz University (KAU), Jeddah, Saudi Arabia. Her current research interests: 5G, Wireless Communications, Wireless Sensor Networks, Internet of Things (IoT) Platform, Internet of Everything (IoE), Connected Homes, Smart Workspace, Machine Learning, Deep Learning, Deep Reinforced Learning, Artificial General Intelligence, Artificial Intelligence Everywhere, Autonomous Vehicles, providing connectivity of IoT devices and sensors for Smart Cities, Location Awareness. During the summers of 2013 and 2014, Dr. Aldabbagh joined Professor John M. Cioffi's Dynamic Spectrum Management (DSM) Research Group as a visiting professor at Stanford University. She was awarded by KAU four awards of excellence in scientific publishing for faculty members for the academic year 2015-2016. In March 2016, Dr. Aldabbagh joined the Laboratory for Information and Decision Systems (LIDS) at Massachusetts Institute of Technology (MIT) as a postdoctoral associate and from March 2017 as a visiting scholar to work on the topic of location-aware ad-hoc sensor networks to work with the highly cited Professor Moe Win. In collaboration with Professor Win's research group, her focus is to develop scalable, distributed, and energy-efficient techniques for scheduling and routing in ad hoc sensor networks with localization-awareness. In November 2019, she participated in the Saudi Think Tank 20 (T20) of the Saudi G20, as a co-author for two policy briefs (PB); the first was in Task Force 10 (TF10): Sustainable Energy, Food and Water Systems and the second PB was TF6: Economy, Employment, And Education In The Digital Age. In April 2020, Dr. Aldabbagh joined the Marconi Society as a member of the Marconi Selection Advisory Committee for the Marconi Prize. 